\title{
Functionalization of Graphene by Atmospheric Pressure Plasma Jet in Air or $\mathrm{H}_{2} \mathrm{O}_{2}$ environments
}

\author{
Weixin Huang ${ }^{1,2}$ and Sylwia Ptasinska ${ }^{1,3 *}$ \\ ${ }^{1}$ Radiation Laboratory, ${ }^{2}$ Department of Chemistry and Biochemistry, and ${ }^{3}$ Department of Physics, \\ University of Notre Dame, Notre Dame, IN 46556, USA
}

\begin{abstract}
The functionalization of graphene, which deforms its band structure, can result in a metal-semiconductor transition. In this work, we report a facile strategy to oxidize single-layer graphene using an atmospheric pressure plasma jet (APPJ) that generates a variety of reactive plasma species at close to ambient temperature. We systematically characterized the oxygen content and chemical structure of the graphene films after plasma treatment under different oxidative conditions (ambient air atmosphere or hydrogen peroxide solution) by X-ray Photoelectron Spectroscopy (XPS). Plasma-treated graphene films containing more than $40 \%$ oxygen were obtained in both oxidative environments. Interestingly, prolonged irradiation led to the reduction of graphene oxides. N-doping of graphene also occurred during the APPJ treatment in $\mathrm{H}_{2} \mathrm{O}_{2}$ solution; the nitrogen content of the doped graphene was dependent on the duration of irradiation and reached up to $8.1 \%$ within 40 minutes. Moreover, the $\mathrm{H}_{2} \mathrm{O}_{2}$ solution served as a buffer layer that prevented damage to the graphene during plasma irradiation. Four-point probe measurement revealed an increase in sheet resistance of the plasma-treated graphene, indicating the transition of the material property from semi-metallic to semiconducting.
\end{abstract}

Keywords: single-layer graphene, functionalization, nitrogen-doped, atmospheric plasma irradiation, graphene reduction

* corresponding author: Sylwia.Ptasinska.1@nd.edu 


\section{Introduction}

Graphene, a two-dimensional $\mathrm{sp}^{2}$-hybridized carbon, has received significant attention due to its unusual physical and chemical properties, which make it a potential material for the fabrication of electronic and optical devices such as sensors and transistors, and for use in catalysis.[1-3] Despite graphene's potential in these applications, the fact that it has a zero band gap and is chemically inert diminish its competitive strength as a semiconducting material.[4] However, the band gap in graphene can be opened and controlled by direct contact with other materials, i.e., i) covalent and non-covalent interactions with inorganic $(\mathrm{O}, \mathrm{OH}, \mathrm{H}$, and $\mathrm{F})$ and organic species, [5-7] and ii) substitutional doping, in which carbon atoms from a hexagonal honeycomb lattice are substituted by nitrogen or boron atoms.[8-14] The oxidation of graphene, for example, breaks the $\mathrm{sp}^{2}$ structure and yields a finite electronic band gap due to the formation of oxygenated functional groups, thus offering tremendous opportunities for access to functionalized graphene-based materials.[6] Alternatively, nitrogen-doped graphene has been reported to improve electron density, Fermi level $\left(\mathrm{E}_{\mathrm{f}}\right)$ tunability, and chemical reactivity. $[8,15]$ When lone pair electrons are conjugated with the graphitic $\pi$-system, doping graphene with nitrogen atoms results in electron-rich graphene sheets, from which semiconducting behavior is expected.

There are many possible ways to achieve graphene functionalization, and the most common methods for introducing atoms or molecules to graphene are annealing at high temperatures, $[9,16]$ chemical vapor deposition (CVD),[17] arc discharge,[18] and solvo-thermal synthesis.[19,20] Plasma treatment is also an effective and economical process for graphene functionalization.[20-22] By using different feed gases in plasmas, various species, such as oxygen, fluorine, and nitrogen, can be grafted to the graphene surface. Previous research has focused on the plasma doping of graphite or multilayer graphene, and has revealed that ion bombardment leads to unavoidable surface damage and ultimately destroys the graphene structure.[23,24] Therefore, the deposition of aluminum oxides on graphene has been proposed to decrease plasma damage.[11]

Compared to other types of plasma treatment, an atmospheric pressure plasma jet (APPJ) has the significant advantage of avoiding both costly complex vacuum systems and high temperatures, and thus can offer many opportunities for applications at the interface of plasma physics, radiation chemistry, and biomedicine.[25] An APPJ consists of multiple types of reactive species, such as radicals, electrons, photons, and charged particles, all of which can be delivered to and interact with samples. In this study, single-layer graphene films on a quartz substrate were treated in air and in an $\mathrm{H}_{2} \mathrm{O}_{2}$ solution by a helium APPJ for various lengths of time. The APPJ has not been used for graphene functionalization before; however it was used recently for silicon surface modification[26]. The oxygen- and nitrogen-containing functional groups of graphene were confirmed and quantified by XPS. The geometrical and electronic configurations of the APPJ source have been reported previously.[26,27]

\section{Experimental}

The experimental setup of the APPJ source has been reported previously.[26,27] Plasma was ignited between two tubular brass electrodes fastened to a fused silica tube (o.d. $6 \mathrm{~mm}$, i.d. $5 \mathrm{~mm}$ ) through 
which helium flowed. The gas flow rate was kept constant at $4 \mathrm{~L} / \mathrm{min}$. The amplitudes of voltage and current on the grounded electrode were recorded as $10.7 \mathrm{kV}$ and $14 \mathrm{~mA}$, respectively. We used a pulse waveform generator (80 MHz Function/Arbitrary Waveform Generator, 33250A, Agilent Tech) to generate a $100 \mu \mathrm{s}$ in width square pulse with a repetition rate of $1.5 \mathrm{kHz}$. The dielectric barrier discharge was projected as a jet with a tip at a distance of a few centimeters from the tube orifice. We note that the luminous part of the APPJ is $\sim 6 \mathrm{~cm}$.

Single-layer graphene on quartz was purchased from Graphenea, USA. Prior to plasma treatment, the graphene sample was heated to $500{ }^{\circ} \mathrm{C}$ for 5 minutes under vacuum conditions $\left(10^{-6} \mathrm{Torr}\right)$ to remove contaminants and reduce the graphene, as the sample was oxidized slightly in air. Then, the sample was cooled to room temperature and removed to ambient conditions. The graphene samples were then treated in air or $50 \mathrm{wt} \% \mathrm{H}_{2} \mathrm{O}_{2}$ conditions. The samples irradiated by plasma in air were defined as graphene-A (GA) and were treated directly with the APPJ for various durations. Under $\mathrm{H}_{2} \mathrm{O}_{2}$ conditions, the samples represented as graphene-B (GB) were immersed in a vial $(20 \mu \mathrm{L}$ max. vol., i.d. $4.5 \mathrm{~mm}$ ). The thickness of the liquid above the sample was kept between $1-1.5 \mathrm{~mm}$ during the plasma irradiaton; the $\mathrm{H}_{2} \mathrm{O}_{2}$ solution was pipetted out after irradiation and the samples were dried before analysis. In both cases, the samples were placed on an adjustable sample holder so that the distance of the sample from the jet could be varied. The graphene samples were characterized by XPS, which was carried out with a PHI VersaProbe II spectrometer ( $\mathrm{Al} \mathrm{K \alpha}$ ). All photoelectron spectra were analyzed using CasaXPS software. The $\mathrm{C} 1 \mathrm{~s}$ signal of the oxidized graphene film consisted of five peaks that corresponded to $\mathrm{sp}^{2}$ carbon at $284.3 \mathrm{eV}, \mathrm{sp}^{3}$ carbon/C-OH at $285.3 \mathrm{eV}$, epoxy $(\mathrm{O}-\mathrm{C}-\mathrm{O})$ at $286.3 \mathrm{eV}$, carbonyl $(\mathrm{C}=\mathrm{O})$ at $288.0 \mathrm{eV}$, and carboxyl $(\mathrm{O}-\mathrm{C}=\mathrm{O})$ at $289.0 \mathrm{eV}$. These assignments were consistent with other reports of graphene oxides.[28-30] The peaks with binding energies at 398.6, 399.9, and $401.8 \mathrm{eV}$ in the $\mathrm{N} 1 \mathrm{~s}$ spectra represent pyridinic, pyrrolic, and quaternary nitrogen, respectively.[10,11] We used a conventional four-point probe (Keithley 2450) to measure sheet resistance.

\section{Results and Discussion}

Figure 1a shows the carbon 1s (C 1s) spectra recorded from graphene films after APPJ exposure in air at ambient temperature. In pristine graphene (GA-0s), the peak at $284.3 \mathrm{eV}$ is due to the main bonding configuration of carbon atoms $\left(\mathrm{C}-\mathrm{C}\left(\mathrm{sp}^{2}\right)\right)$. The $\mathrm{sp}^{3}$-hybridized carbons (C-C $\left(\mathrm{sp}^{3}\right)$ ) bearing hydroxyl functional groups are correlated with the hydroxyl $(\mathrm{C}-\mathrm{OH})$ peak at $285.3 \mathrm{eV}$. After plasma irradiation, new oxygenated components (epoxy (C-O-C), carbonyl $(\mathrm{C}=\mathrm{O})$, and carboxyl $(\mathrm{O}-\mathrm{C}=\mathrm{O})$ ) developed gradually in the $\mathrm{C}$ 1s spectra at higher binding energies. The relative percentages of the oxygen-containing species, as determined from the $\mathrm{C} 1 \mathrm{~s}$ spectra, are plotted in Figure $1 \mathrm{~b}$. As irradiation time increased to 1 minute, the epoxy fraction increased from approximately 6 to 34\%, and the fraction of all other oxygenated functional groups increased up to $40 \%$. We note that the process of carbonyl and carboxyl group formation occurred very rapidly: an APPJ exposure of only 10 seconds resulted in the formation of $2.5 \%$ of $\mathrm{C}=\mathrm{O}$ and $5 \%$ of $\mathrm{O}-\mathrm{C}=\mathrm{O}$, and their abundance remained constant over a range of irradiation times. After 1 minute of treatment, the C-O-C group clearly became the dominant fraction (> 35\%) of the total oxygenated functional groups formed on the sample. It is obvious that graphene films are oxidized easily by a plasma jet, indicating that APPJ reactive species oxidize graphene by attacking the carbon $\mathrm{sp}^{2}$ structure directly and develop the oxygenated functional groups in graphene gradually. 
(a)

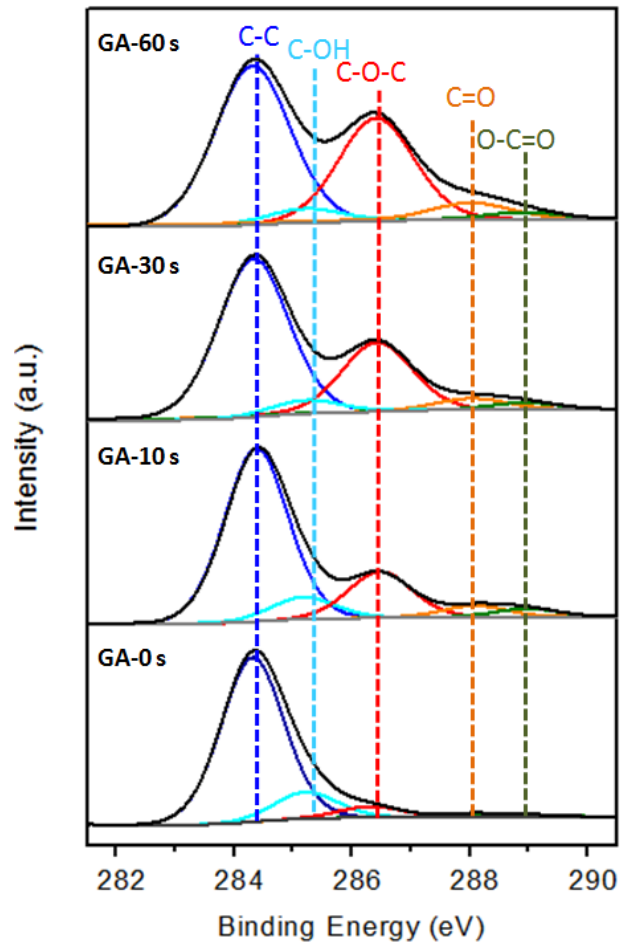

(b)

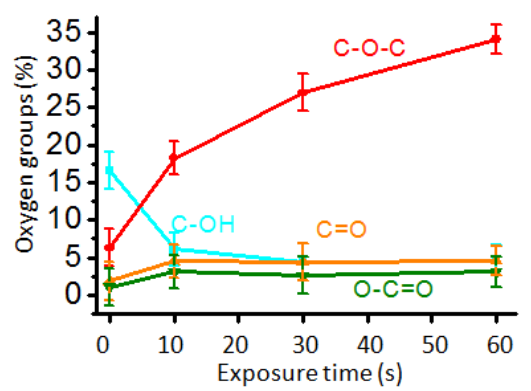

(c)

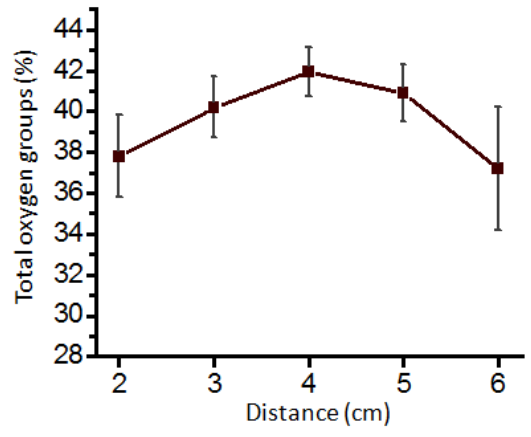

Figure 1. (a) C 1s XPS profiles of APPJ-treated graphene films exposed to air (defined as graphene-A (GA)) for various irradiation times.; (b) concentration of oxygenated functional groups as a function of time, and (c) as a function of distance from the tube orifice. The distance between the tube orifice and graphene samples was maintained at $4 \mathrm{~cm}$ in (a) and (b).

The second variable we explored with respect to the APPJ interactions with graphene was the distance between the sample and the tube orifice, as the distribution of the reactive species in the plasma jet varies along its length.[27,31,32] In order to determine the most effective position for plasma irradiation, we examined the surface oxygen-containing species with respect to the distance between the tube orifice and the sample. Figure 1c shows the fraction of the total percentage of oxygenated functional groups as a function of the distance from the orifice with 1 minute of plasma treatment. The fraction of oxygen-containing species exceeded $40 \%$ at a distance of $4 \mathrm{~cm}$. If the sample distance was increased beyond $4 \mathrm{~cm}$, however, the total percentage of the oxygenated functional groups decreased. This decrease was due to the diffusion of radicals into the atmosphere at greater distances from the APPJ.

APPJ irradiation was also performed in the air for extended periods of time ( $>1$ minute). As the duration of exposure increased, the epoxy peak at $286.3 \mathrm{eV}$ decreased, as shown in Figure 2a. The fraction of epoxy species declined, which can be partially attributed to the atomic rearrangement and conversion of epoxy to carbonyl and/or carboxyl forms with plasma treatment.[33] However, the overall oxygen content $(\mathrm{C}-\mathrm{OH}, \mathrm{C}-\mathrm{O}-\mathrm{C}, \mathrm{C}=\mathrm{O}$, and $\mathrm{O}-\mathrm{C}=\mathrm{O})$ in single-layer graphene film decreased, indicating that APPJ leads to a reduction of graphene oxide in ambient air conditions. It has been reported that a low energy $\mathrm{Ar}^{+}$beam can be employed to reduce graphene oxide into graphene accompanied by the formation of $\mathrm{CO}, \mathrm{CO}_{2}$, and $\mathrm{H}_{2} \mathrm{O}$.[34] Localized heating from ion 
energy results in the decomposition of oxygen functionalities. The energy transferred from ions causes local heating of the graphene oxide, which can be followed by oxygen functional group decomposition. A similar effect was not likely to be observed in this study with respect to oxygen reduction by APPJ treatment due to the collisional energy loss of ions at atmospheric pressure. However, other processes such as energy transfer from excited metastable species can be responsible for the oxygen reduction. In addition, an etching effect also accompanied the reduction oxygen reduction by long-term APPJ treatment. We estimated the amount of graphene remaining on the quartz substrate by calculating the ratio of carbon to the sum of carbon and silicon atoms. This ratio was calculated as the integrated area of the $\mathrm{C} 1 \mathrm{~s}$ peak and the sum of the areas of the $\mathrm{C}$ $1 \mathrm{~s}$ and Si $2 \mathrm{p}$ peaks, and took into account their corresponding XPS sensitivity factors. Figure $2 \mathrm{c}$ shows the atomic ratio $(\mathrm{C} 1 \mathrm{~s} /(\mathrm{C} 1 \mathrm{~s}+\mathrm{Si} 2 \mathrm{p}))$ as a function of plasma treatment, indicating that the single-layer graphene sample remained nearly intact after 1 minute of irradiation. However, the $\mathrm{C} 1 \mathrm{~s} /(\mathrm{C} 1 \mathrm{~s}+\mathrm{Si} 2 \mathrm{p})$ ratio was found to be half than the initial ratio after 2 minutes of irradiation, indicating that the etching phenomenon occurs during long exposures to the APPJ.
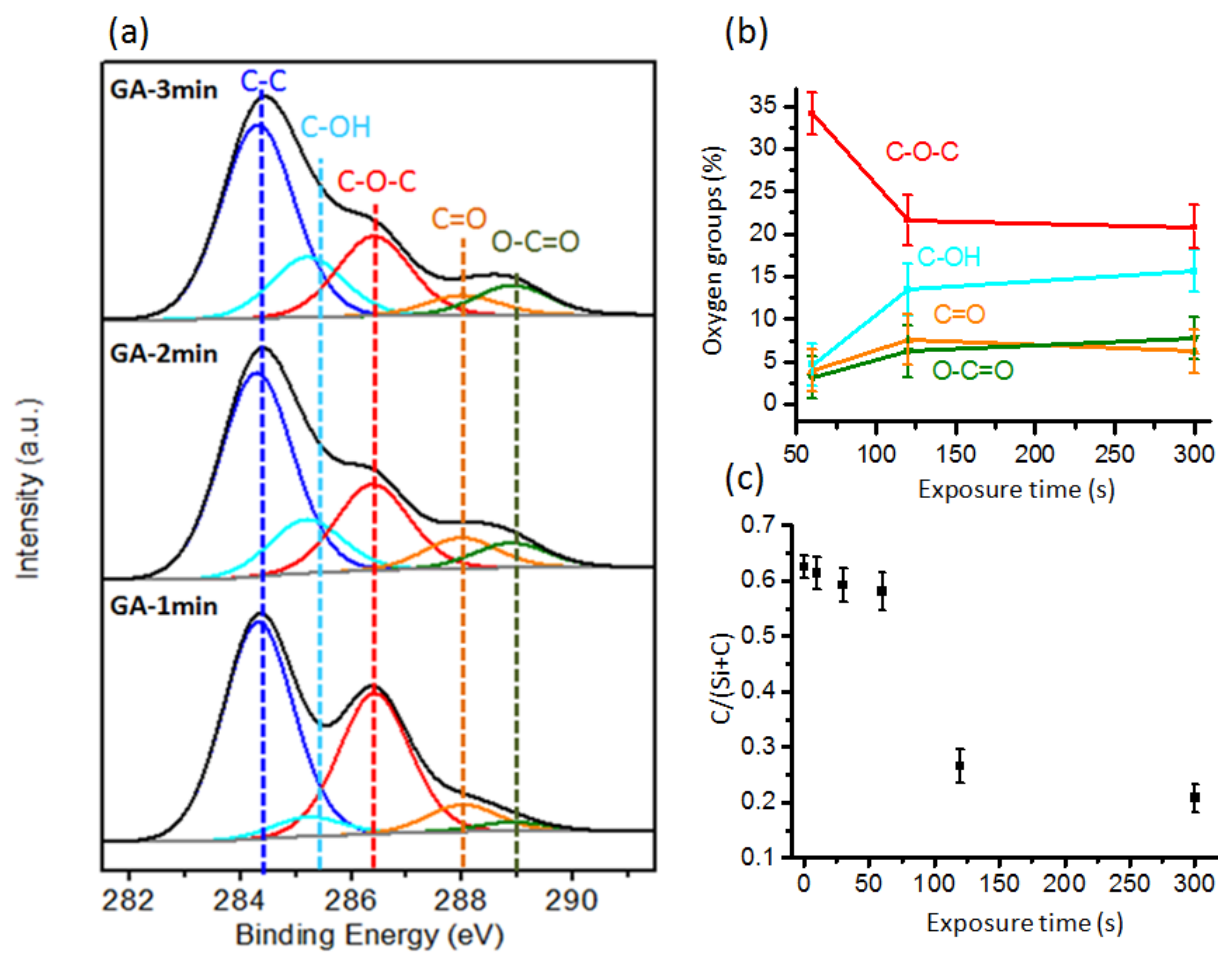

(c)

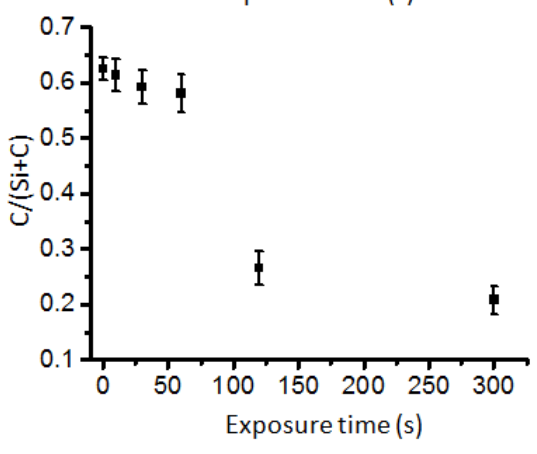

Figure 2. (a) C1s XPS profiles of APPJ-treated graphene films exposed to air conditions for various irradiation times; (b) concentration of oxygenated functional groups, and (c) the ratio of carbon to the sum of carbon and silicon as a function of exposure time.

The same experimental procedure was also carried out for graphene samples immersed in an $\mathrm{H}_{2} \mathrm{O}_{2}$ solution. Figure 3a displays the $\mathrm{C} 1 \mathrm{~s}$ spectra obtained from various durations of APPJ treatment. Peak fitting of the $\mathrm{C} 1 \mathrm{~s}$ peak revealed the presence of and gradual increase in oxygenated functional groups $(\mathrm{C}-\mathrm{O}-\mathrm{C}, \mathrm{C}=\mathrm{O}$, and $\mathrm{O}-\mathrm{C}=\mathrm{O}$ ). It is important to note that the $\mathrm{C} 1 \mathrm{~s}$ spectrum obtained from the sample, which was immersed in the $\mathrm{H}_{2} \mathrm{O}_{2}$ solution for 40 minutes, showed no significant changes in comparison to the spectra obtained for pristine graphene. However, in a 
comparison between two different oxidative conditions, we note that oxygen-containing species were produced at shorter exposure times in air (10 s, in Figure 1a) because a larger number of reactive species, such as $\mathrm{OH}, \mathrm{O}$, and $\mathrm{H}_{3} \mathrm{O}^{+}$, could reach the graphene directly. In contrast, for samples immersed in solution, other reactive species, such as $\bullet \mathrm{OH}$, and $\mathrm{O}_{2}^{-}$, can also be formed at the plasma-liquid interface.[35,36] However, it is comparatively more difficult for these to diffuse in order to reach the graphene. The total oxygen content in the plasma-treated graphene films after 20 minutes also reached $40 \%$, whereas longer durations of plasma irradiation resulted in the reduction of oxygenated functional groups, as shown in Figure 3b. In addition, we observed no etching effect from possible damage by ions in the APPJ, even over a wide range of APPJ treatments (Figure 3c). The $\mathrm{H}_{2} \mathrm{O}_{2}$ solution reduced the kinetic energy of the plasma ions sufficiently due to their inelastic scattering. Thus, the liquid phase functions as a buffer layer that effectively prevents ions from destroying the graphene during the plasma treatment.
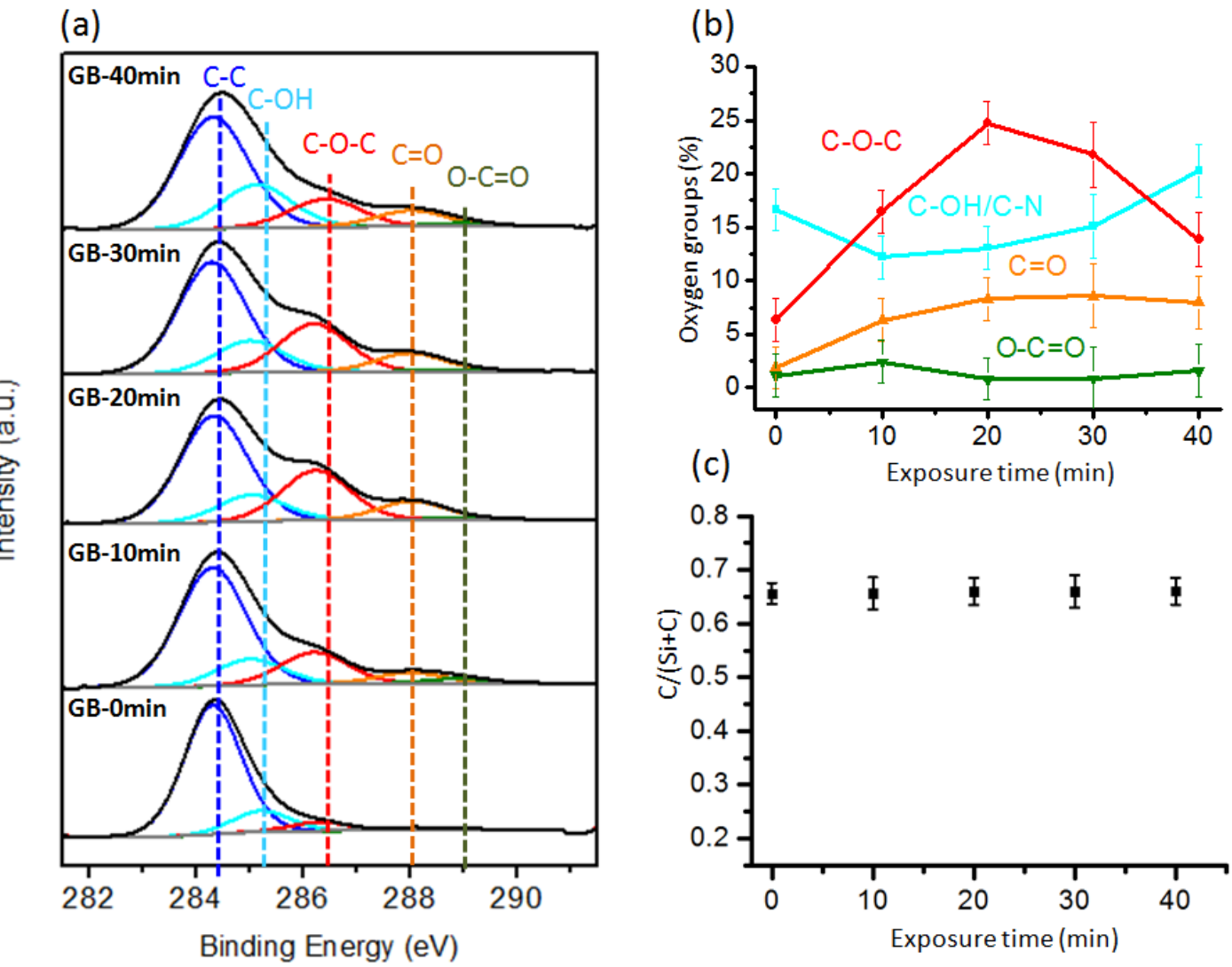

Figure 3. (a) $\mathrm{C} 1 \mathrm{~s}$ XPS profiles of APPJ-treated graphene films immersed in 50wt $\% \mathrm{H}_{2} \mathrm{O}_{2}$ solution (defined as graphene-B (GB)) for various irradiation times; (b) concentration of oxygenated functional groups, and (c) the ratio of carbon to the sum of carbon and silicon as a function of exposure time.

The formation of oxygenated functional groups $(\mathrm{C}-\mathrm{O}-\mathrm{C}, \mathrm{C}=\mathrm{O}$, and $\mathrm{O}-\mathrm{C}=\mathrm{O})$ represents the total extent of the graphene oxidation under air and $\mathrm{H}_{2} \mathrm{O}_{2}$ conditions. Therefore, the oxidation rate can be determined as the oxidation fraction within the total exposure time. Figure 4 shows the percentage of oxygenated functional groups formed under air and $\mathrm{H}_{2} \mathrm{O}_{2}$ conditions as a function of plasma exposure time. We generated a maximum-likelihood linear fit to the data in order to compare the graphene oxidation rates in these two oxidative environments. The oxidation rates 
were estimated to be 0.50 and $0.02 \mathrm{~s}^{-1}$ in air and $\mathrm{H}_{2} \mathrm{O}_{2}$ solution, respectively, indicating that the oxidation of graphene can be achieved much more rapidly in air.

(a)

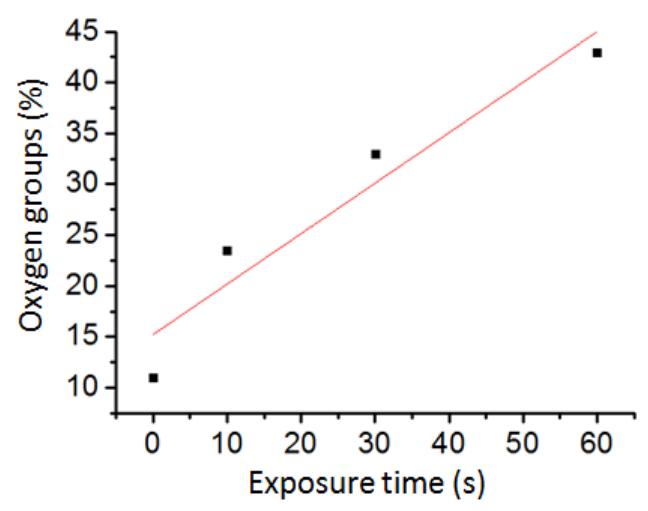

(b)

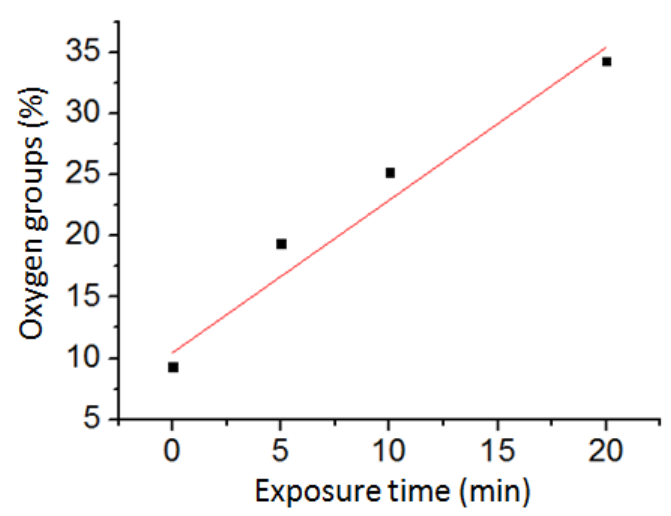

Figure 4. (a) Concentration of oxygenated functional groups (C-O-C, $\mathrm{C}=\mathrm{O}$, and $\mathrm{O}-\mathrm{C}=\mathrm{O}$ ) formed on the graphene in air, and (b) in $\mathrm{H}_{2} \mathrm{O}_{2}$ solution as a function of APPJ exposure time.

Interestingly, we also observed that $\mathrm{N}$-atoms were substitutionally doped into the graphene films immersed in the $\mathrm{H}_{2} \mathrm{O}_{2}$ solution and irradiated by the APPJ. Survey spectra (Figure 5a) of the graphene samples before and after plasma irradiation show differences in their atomic composition and indicate the presence of nitrogen atomic species. The Sn 3d peaks visible in the survey spectrum originated from the stabilizer of the $\mathrm{H}_{2} \mathrm{O}_{2}$ solution. The quantity of nitrogen incorporated into the graphene was estimated by XPS using the integrated area of the $\mathrm{N} 1 \mathrm{~s}$ and $\mathrm{C} 1 \mathrm{~s}$ peaks and taking into consideration the relative sensitivity factors. Figure $5 \mathrm{~b}$ shows the atomic percentage of nitrogen incorporated into graphene as a function of plasma treatment time. The nitrogen introduced during the plasma treatment was found to comprise $8.5 \%$ of the material. In contrast, no nitrogen peak was observed in the XPS spectra of GA samples. This is because oxidation was still predominant in comparison to $\mathrm{N}$-doping during the first 2 minutes. To evaluate the chemical states and bonding configurations of doped nitrogen atoms, we examined the $\mathrm{N} 1 \mathrm{~s}$ spectrum obtained from the GB-20min (Figure 5c). As seen in Figure 5d, quaternary nitrogen, which is known as "graphitic nitrogen", was incorporated into the graphene layer and substituted carbon atoms; this is also known as a p-doping effect.[8] The $\mathrm{N}$ atoms in pyridinic $\mathrm{N}$ were bonded to two carbon atoms, which donated one p-electron to the $\pi$ system. Pyrrolic $\mathrm{N}$ refers to $\mathrm{N}$ atoms incorporated into five-membered heterocyclic rings. This contributed two p-electrons to the $\pi$ system. Eventually, both pyridinic and pyrrolic $\mathrm{N}$ atoms can increase the $\mathrm{E}_{\mathrm{f}}$ and density of states of graphene.[37,38] The nitric/nitridic ions $\left(\mathrm{NO}_{2}{ }^{-} / \mathrm{NO}_{3}{ }^{-}\right)$formed at the plasma-liquid interface are known to be major nitrogen species.[35] Burgess et al.[39] reported that an amine structure (with binding energy of $400.2 \mathrm{eV}$ in $\mathrm{N} 1 \mathrm{~s}$ ) and hydroxyl groups were detected in graphite samples after 30 minutes of washing with nitric acid. In our case, reactive species initiated by APPJ, such as $\mathrm{NO}_{2}{ }^{-}$and $\mathrm{NO}_{3}{ }^{-}$, can diffuse into solution and react with graphene to form three types of N-doping structures. In the case of graphene in $\mathrm{H}_{2} \mathrm{O}_{2}$ solution treated by APPJ, graphene-based derivatives (oxygen- and nitrogen-containing species) could be produced, and depending on the amount of doped elements or bonded molecules, it is also possible to tune the electronic properties precisely 
for industrial applications such as sensors, and energy conversion and storage.

(a)

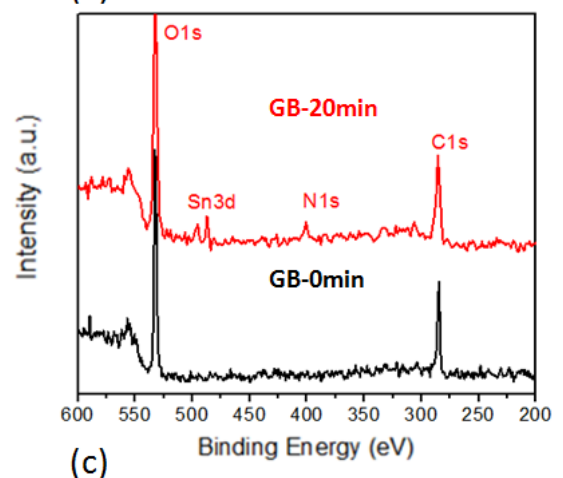

(c)

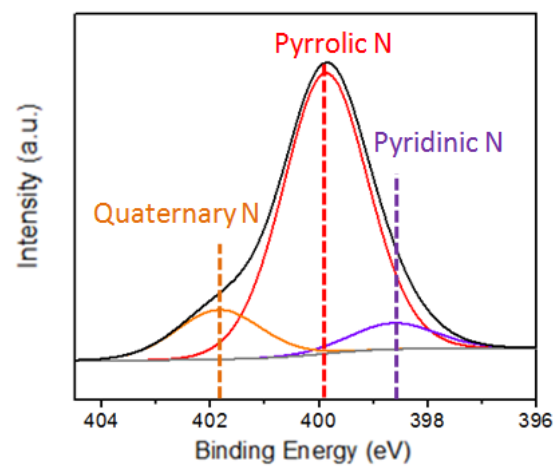

(b)

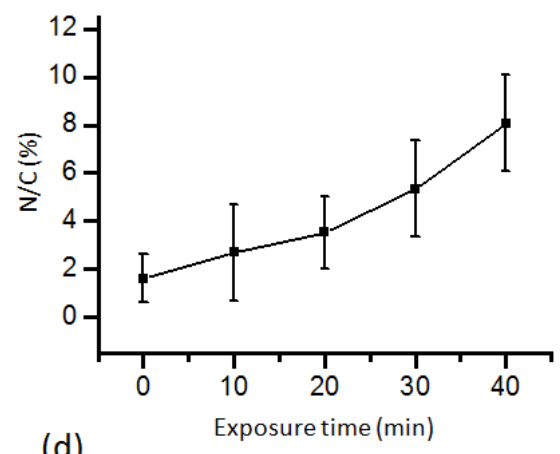

(d)

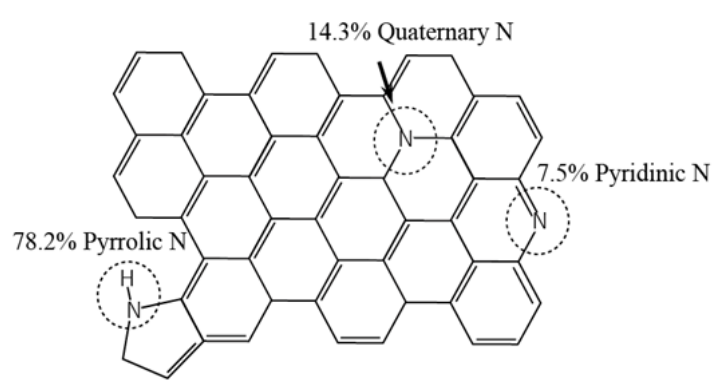

Figure 5. (a) Survey spectra of graphene (GB-0 min) and N-doped graphene (GB-20 min), (b) content of nitrogen atoms of $\mathrm{N}$-graphene as a function of the plasma treatment time, (c) N 1s XPS profile showing the contribution from different nitrogen species, and (d) schematic of postulated nitrogen structure in graphene.

To gain insight into the electrical properties of this material, we measured sheet resistance as a function of APPJ exposure time (Figure 6) using the Van der Pauw method[40,41]. For graphene samples treated by APPJ in air, the sheet resistance increased with exposure time and reached 780 $\mathrm{k} \Omega / \mathrm{sq}$ after 1 minute of irradiation. The enhancement in sheet resistance indicated the opening of the bandgap, thus the observed transition from semi-metallic to semiconducting behavior of the graphene.[6] For graphene samples treated in $\mathrm{H}_{2} \mathrm{O}_{2}$ solution for 20 minutes, the sheet resistance increased to $573 \mathrm{k} \Omega / \mathrm{sq}$. In contrast, for longer periods (40 minutes) of APPJ exposure, sheet resistance decreased due to the substitution of oxygen species by the nitrogen groups. It is known that $\mathrm{N}$-doped graphene has lower sheet resistance than graphene oxide.[9] Nevertheless, the high sheet resistance in both cases can be collectively accounted for by the high oxygen/nitrogen content, which is consistent with the previously reported sheet resistance of functionalized graphene.[6,9] 

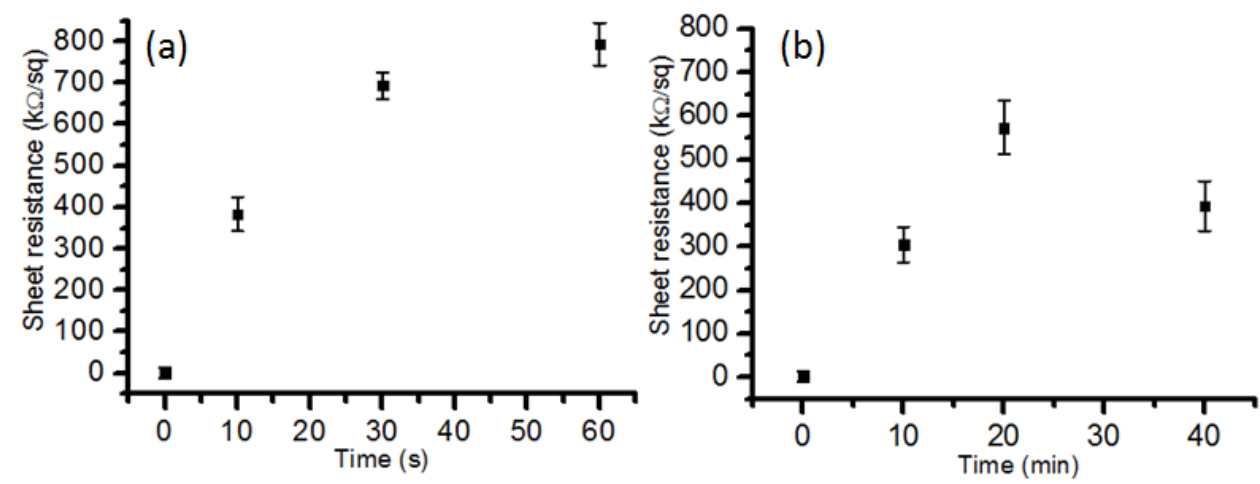

Figure 6. Sheet resistance of graphene treated by APPJ (a) in air and (b) in $\mathrm{H}_{2} \mathrm{O}_{2}$ solution as a function of time.

Understanding the growth of oxygen-containing species in response to plasma irradiation is central to the development of strategies for achieving controlled functionalization of graphene. Figure 7 shows a schematic of possible mechanisms of the growth and reduction of oxygen species on graphene in ambient air conditions. Initially, atomic oxygen from plasma irradiation is bonded into graphene and become epoxy groups, which is shown in step (1). This occurs because epoxy groups are unstable under APPJ irradiation and can be bombarded by plasma electrons and then dissociate. Then, a reactive oxygen species $(\mathrm{C}-\mathrm{O} \bullet)$ is formed from the epoxy group (C-O-C) following the breaking of one of the $\mathrm{C}-\mathrm{O}$ bonds. After the activation of $\mathrm{C}-\mathrm{O}-\mathrm{C}$, the resulting transient surface atomic oxygen is captured immediately by the surrounding oxygen, forming a di-oxygen molecule as the product (occurring in steps (2)). For graphene with a high epoxy species coverage, we expect that the rate of reduction would exceed that of oxidation, such that the graphene would be reduced until the forward and reverse reactions achieve thermodynamic equilibrium.

Similarly, graphene oxidation in the $\mathrm{H}_{2} \mathrm{O}_{2}$ solution is illustrated in the initial in Figure 7. However, the liquid acts as a protection layer and prevents the reduction of graphene oxide by electron/ion bombardment. Therefore, the reduction is primarily attributed to N-doping, in which graphene oxides react with $\mathrm{N}$-containing species in the liquid (occurring in steps $(3)$ ). $[9,10,42]$ 


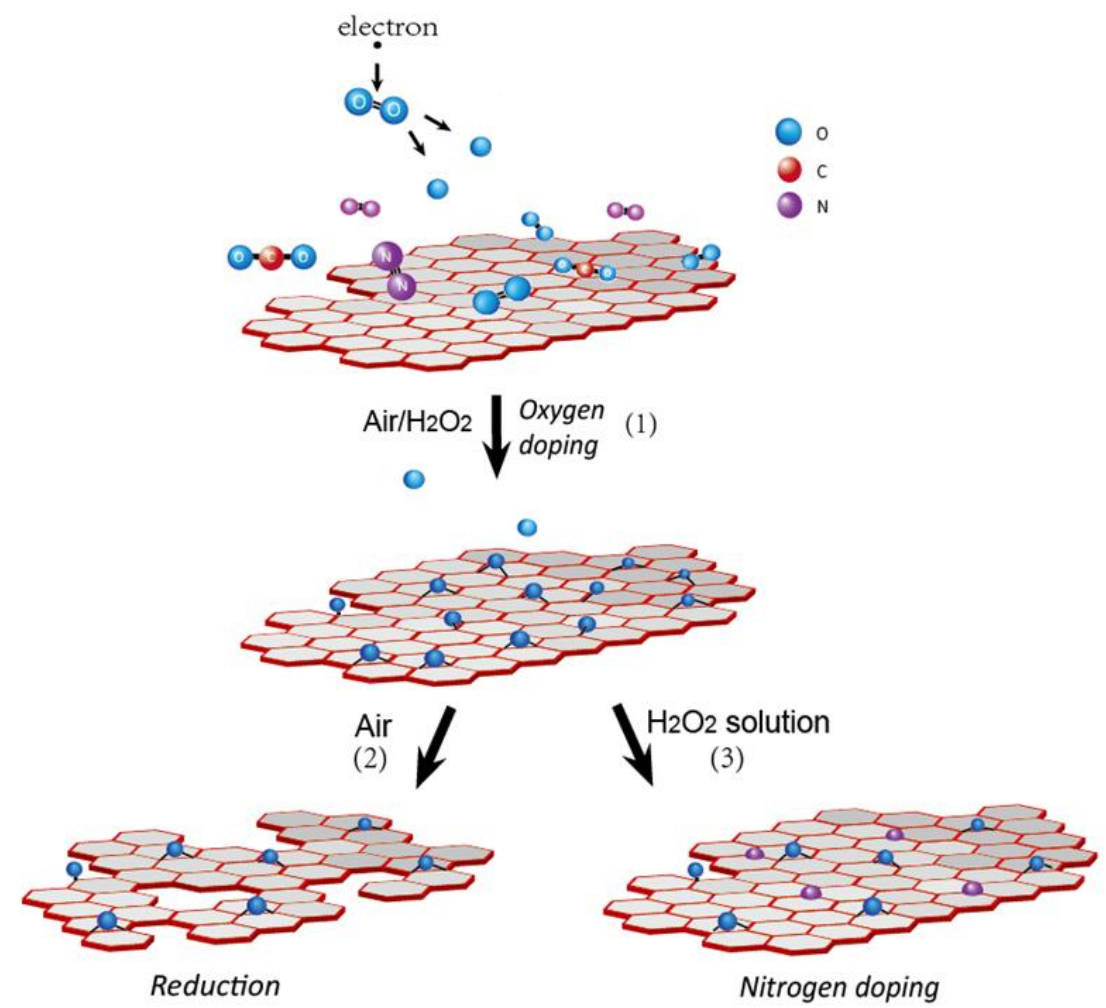

Figure 7. Schematics of plasma-induced processes.

\section{Conclusions}

We have demonstrated a facile plasma approach for functionalizing graphene in air and in $\mathrm{H}_{2} \mathrm{O}_{2}$ solution. By controlling the duration of irradiation, we can change the atomic oxygen content in graphene by up to $40 \%$. Further irradiation caused both etching and reduction of oxidized graphene and this is the first time a reduction of graphene has been observed in oxidative surroundings. Etching appeared in the ambient air conditions within 2 minutes after the onset of APPJ treatment, but did not do so in the $\mathrm{H}_{2} \mathrm{O}_{2}$ solution. We noted as well that $\mathrm{N}$-doped graphene was produced, and $8.2 \%$ nitrogen was substitutionally doped into the graphene. In addition, plasma-treated graphene exhibited a typical resistance in the range of $500-780 \mathrm{k} \Omega / \mathrm{sq}$, indicating the extent of the bandgap opening upon graphene functionalization. This facile synthesis of functionalized (O- and $\mathrm{N}$-doped) graphene by APPJs could be of great benefit in the production of semiconductors and other emerging energy materials.

\section{Acknowledgements}

This material is based upon work supported by the U.S. Department of Energy, Office of Science, Office of Basic Energy Sciences under Award Number DE-FC02-04ER15533. This is contribution number NDRL 5072 from the Notre Dame Radiation Laboratory. W.H. thanks the cSEND Materials Characterization Facility for the use of the PHI VersaProbe II XPS. W.H. acknowledges D. Sokolov for their fruitful discussions, and E.R. Adhikari and X. Han for their technical assistance during the initial stage of the project. 


\section{References}

[1] K.S. Novoselov, V.I. Fal'ko, L. Colombo, P.R. Gellert, M.G. Schwab, K. Kim, A roadmap for graphene., Nature. 490 (2012) 192-200. doi:10.1038/nature11458.

[2] F. Bonaccorso, A. Lombardo, T. Hasan, Z. Sun, L. Colombo, A.C. Ferrari, Production and processing of graphene and 2d crystals, Mater. Today. 15 (2012) 564-589. doi:10.1016/S1369-7021(13)70014-2.

[3] X. Li, G. Zhang, X. Bai, X. Sun, X. Wang, E. Wang, et al., Highly conducting graphene sheets and Langmuir-Blodgett films., Nat. Nanotechnol. 3 (2008) 538-42. doi:10.1038/nnano.2008.210.

[4] V. Georgakilas, M. Otyepka, A.B. Bourlinos, V. Chandra, N. Kim, K.C. Kemp, et al., Functionalization of graphene: covalent and non-covalent approaches, derivatives and applications., Chem. Rev. 112 (2012) 6156-214. doi:10.1021/cr3000412.

[5] J. Park, W.H. Lee, S. Huh, S.H. Sim, S. Bin Kim, K. Cho, et al., Work-Function Engineering of Graphene Electrodes by Self-Assembled Monolayers for High-Performance Organic Field-Effect Transistors, J. Phys. Chem. Lett. 2 (2011) 841-845. doi:10.1021/jz200265w.

[6] A. Nourbakhsh, M. Cantoro, T. Vosch, G. Pourtois, F. Clemente, M.H. van der Veen, et al., Bandgap opening in oxygen plasma-treated graphene., Nanotechnology. 21 (2010) 435203. doi:10.1088/0957-4484/21/43/435203.

[7] R. Balog, B. Jørgensen, L. Nilsson, M. Andersen, E. Rienks, M. Bianchi, et al., Bandgap opening in graphene induced by patterned hydrogen adsorption., Nat. Mater. 9 (2010) 315-9. doi:10.1038/nmat2710.

[8] X. Wang, X. Li, L. Zhang, Y. Yoon, P.K. Weber, H. Wang, et al., N-doping of graphene through electrothermal reactions with ammonia., Science. 324 (2009) 768-71.

doi:10.1126/science.1170335.

[9] X. Li, H. Wang, J.T. Robinson, H. Sanchez, G. Diankov, H. Dai, Simultaneous nitrogen doping and reduction of graphene oxide., J. Am. Chem. Soc. 131 (2009) 15939-44. doi:10.1021/ja907098f.

[10] K.H. Lee, J. Oh, J.G. Son, H. Kim, S.-S. Lee, Nitrogen-doped graphene nanosheets from bulk graphite using microwave irradiation., ACS Appl. Mater. Interfaces. 6 (2014) 6361-8. doi:10.1021/am405735c.

[11] S.H. Park, J. Chae, M.-H. Cho, J.H. Kim, K.-H. Yoo, S.W. Cho, et al., High concentration of nitrogen doped into graphene using N2 plasma with an aluminum oxide buffer layer, J. Mater. Chem. C. 2 (2014) 933. doi:10.1039/c3tc31773k. 
[12] Z.-H. Sheng, H.-L. Gao, W.-J. Bao, F.-B. Wang, X.-H. Xia, Synthesis of boron doped graphene for oxygen reduction reaction in fuel cells, J. Mater. Chem. 22 (2012) 390. doi:10.1039/c1jm14694g.

[13] M. Xing, F. Shen, B. Qiu, J. Zhang, Highly-dispersed boron-doped graphene nanosheets loaded with TiO2 nanoparticles for enhancing CO2 photoreduction., Sci. Rep. 4 (2014) 6341. doi:10.1038/srep06341.

[14] S. Zhou, D. Siegel, a. Fedorov, a. Lanzara, Metal to Insulator Transition in Epitaxial Graphene Induced by Molecular Doping, Phys. Rev. Lett. 101 (2008) 086402.

doi:10.1103/PhysRevLett.101.086402.

[15] H. Wang, J.T. Robinson, X. Li, H. Dai, Solvothermal reduction of chemically exfoliated graphene sheets., J. Am. Chem. Soc. 131 (2009) 9910-1. doi:10.1021/ja904251p.

[16] S. Wakeland, R. Martinez, J.K. Grey, C.C. Luhrs, Production of graphene from graphite oxide using urea as expansion-reduction agent, Carbon N. Y. 48 (2010) 3463-3470.

doi:10.1016/j.carbon.2010.05.043.

[17] A. Reddy, A. Gowda, Gullapalli, Hemtej, Madan, Dubey, Pulickel, Synthesis Of Nitrogen-Doped Graphene Films For Lithium Battery Application, ACS Nano. 4 (2010) 6337-6342.

[18] K. Gong, F. Du, Z. Xia, M. Durstock, L. Dai, Nitrogen-doped carbon nanotube arrays with high electrocatalytic activity for oxygen reduction., Science. 323 (2009) 760-764.

doi:10.1126/science.1168049.

[19] B.T. McGrail, B.J. Rodier, E. Pentzer, Rapid Functionalization of Graphene Oxide in Water, Chem. Mater. 26 (2014) 5806-5811. doi:10.1021/cm5031409.

[20] P. Zhang, K. Jiang, C. Ye, Y. Zhao, Facile synthesis of V-shaped copolymer brushes grafted onto the surface of graphene oxide via coupling reactions., Chem. Commun. (Camb). 47 (2011) 9504-6. doi:10.1039/c1cc12978c.

[21] J. Senthilnathan, K.S. Rao, M. Yoshimura, Submerged liquid plasma - low energy synthesis of nitrogen-doped graphene for electrochemical applications, J. Mater. Chem. A. 2 (2014) 3332. doi:10.1039/c3ta14946c.

[22] S.W. Lee, C. Mattevi, M. Chhowalla, R.M. Sankaran, Plasma-Assisted Reduction of Graphene Oxide at Low Temperature and Atmospheric Pressure for Flexible Conductor Applications, J. Phys. Chem. Lett. 3 (2012) 772-777. doi:10.1021/jz300080p.

[23] L. Xie, L. Jiao, H. Dai, Selective etching of graphene edges by hydrogen plasma., J. Am. Chem. Soc. 132 (2010) 14751-3. doi:10.1021/ja107071g. 
[24] H.M. Jeong, J.W. Lee, W.H. Shin, Y.J. Choi, H.J. Shin, J.K. Kang, et al., Nitrogen-doped graphene for high-performance ultracapacitors and the importance of nitrogen-doped sites at basal planes., Nano Lett. 11 (2011) 2472-7. doi:10.1021/nl2009058.

[25] T. von Woedtke, S. Reuter, K. Masur, K.-D. Weltmann, Plasmas for medicine, Phys. Rep. 530 (2013) 291-320. doi:10.1016/j.physrep.2013.05.005.

[26] X. Zhang, S. Ptasinska, Growth of silicon oxynitride films by atmospheric pressure plasma jet, J. Phys. D. Appl. Phys. 47 (2014) 145202. doi:10.1088/0022-3727/47/14/145202.

[27] X. Han, W.A. Cantrell, E.E. Escobar, S. Ptasinska, Plasmid DNA damage induced by helium atmospheric pressure plasma jet, Eur. Phys. J. D. 68 (2014) 46. doi:10.1140/epjd/e2014-40753-y.

[28] D. Yang, A. Velamakanni, G. Bozoklu, S. Park, M. Stoller, R.D. Piner, et al., Chemical analysis of graphene oxide films after heat and chemical treatments by $\mathrm{X}$-ray photoelectron and Micro-Raman spectroscopy, Carbon N. Y. 47 (2009) 145-152. doi:10.1016/j.carbon.2008.09.045.

[29] A.I. Aria, M. Gharib, Reversible tuning of the wettability of carbon nanotube arrays: the effect of ultraviolet/ozone and vacuum pyrolysis treatments., Langmuir. 27 (2011) 9005-11. doi:10.1021/la201841m.

[30] A.I. Aria, A.W. Gani, M. Gharib, Effect of dry oxidation on the energy gap and chemical composition of CVD graphene on nickel, Appl. Surf. Sci. 293 (2014) 1-11. doi:10.1016/j.apsusc.2013.11.117.

[31] M. Klas, S. Ptasinska, Characteristics of N 2 and N 2 /O 2 atmospheric pressure glow discharges, Plasma Sources Sci. Technol. 22 (2013) 025013. doi:10.1088/0963-0252/22/2/025013.

[32] B. Bahnev, M.D. Bowden, A. Stypczyńska, S. Ptasińska, N.J. Mason, N.S.J. Braithwaite, A novel method for the detection of plasma jet boundaries by exploring DNA damage, Eur. Phys. J. D. 68 (2014) 140. doi:10.1140/epjd/e2014-40844-9.

[33] J.R. Rani, J. Lim, J. Oh, J. Kim, H.S. Shin, J.H. Kim, et al., Epoxy to Carbonyl Group Conversion in Graphene Oxide Thin Films : E ff ect on Structural and Luminescent Characteristics, (2012) 0-7.

[34] P. Šimek, Z. Sofer, O. Jankovský, D. Sedmidubský, M. Pumera, Oxygen-Free Highly Conductive Graphene Papers, Adv. Funct. Mater. 24 (2014) 4878-4885. doi:10.1002/adfm.201304284.

[35] S. Samukawa, M. Hori, S. Rauf, K. Tachibana, P. Bruggeman, G. Kroesen, et al., The 2012 Plasma Roadmap, J. Phys. D. Appl. Phys. 45 (2012) 253001. doi:10.1088/0022-3727/45/25/253001. 
[36] P. Bruggeman, C. Leys, Non-thermal plasmas in and in contact with liquids, J. Phys. D. Appl. Phys. 42 (2009) 053001. doi:10.1088/0022-3727/42/5/053001.

[37] S.H. Yang, W.H. Shin, J.K. Kang, The nature of graphite- and pyridinelike nitrogen configurations in carbon nitride nanotubes: dependence on diameter and helicity., Small. 4 (2008) 437-41. doi:10.1002/smll.200700543.

[38] B. Biel, X. Blase, F. Triozon, S. Roche, Anomalous Doping Effects on Charge Transport in Graphene Nanoribbons, Phys. Rev. Lett. 102 (2009) 096803. doi:10.1103/PhysRevLett.102.096803.

[39] R. Burgess, C. Buono, P.R. Davies, R.J. Davies, T. Legge, A. Lai, et al., The functionalisation of graphite surfaces with nitric acid: Identification of functional groups and their effects on gold deposition, J. Catal. 323 (2015) 10-18. doi:10.1016/j.jcat.2014.12.021.

[40] L. van der PAUYV, A method of measuring specific resistivity and Hall effect of discs of arbitrary shape, Philips Res. Rep. (1958).

http://faculty.mint.ua.edu/ pleclair/PH255/templates/van_der_Pauw/1958 Van der Pauw (Philips Res Rep) A method of measuring specific resistivity and Hall effect of discs of arbitrary shape.pdf (accessed November 2, 2015).

[41] D. a. Sokolov, C.M. Rouleau, D.B. Geohegan, T.M. Orlando, Excimer laser reduction and patterning of graphite oxide, Carbon N. Y. 53 (2013) 81-89.

doi:10.1016/j.carbon.2012.10.034.

[42] Y. Wang, Y. Shao, D.W. Matson, J. Li, Y. Lin, Nitrogen-doped graphene and its application in electrochemical biosensing., ACS Nano. 4 (2010) 1790-8. doi:10.1021/nn100315s. 


\section{Functionalization of Graphene by Atmospheric Pressure Plasma Jet in Air or $\mathrm{H}_{2} \mathrm{O}_{2}$ environments}

Weixin Huang ${ }^{1,2}$ and Sylwia Ptasinska ${ }^{1,3 *}$

${ }^{1}$ Radiation Laboratory, ${ }^{2}$ Department of Chemistry and Biochemistry, and ${ }^{3}$ Department of Physics, University of Notre Dame, Notre Dame, IN 46556, USA

Graphical Abstract
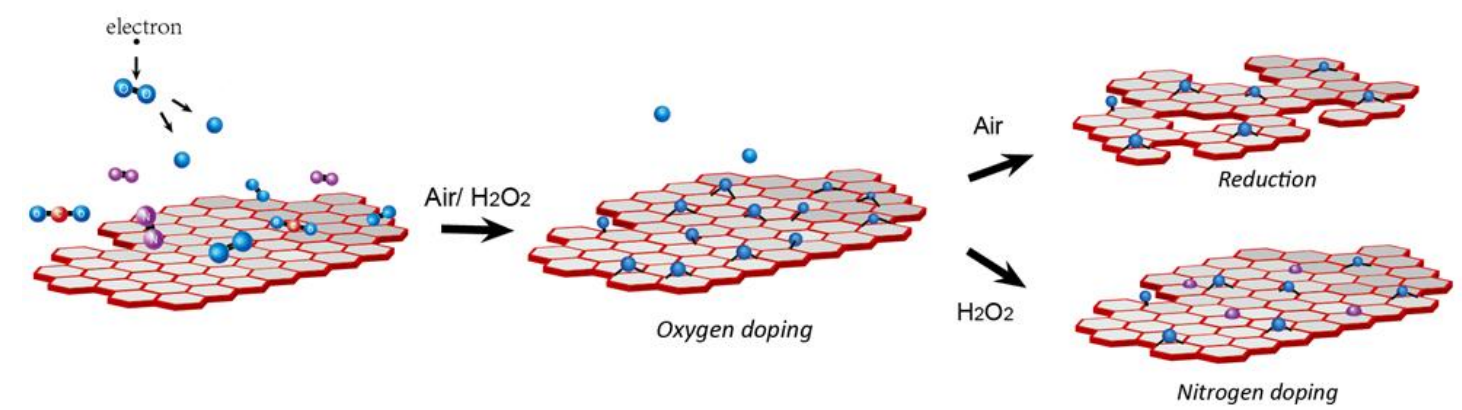